\title{
El lenguaje inclusivo como "norma" de empatía e identidad: reflexiones entre docentes y futures profesores $^{*}$
}

\author{
Silvia Castillo Sánchez ${ }^{* *}$ \\ Simona Mayo ${ }^{* * *}$
}

\section{Resumen}

En el siguiente texto se presenta una reflexión en torno al uso de lenguaje inclusivo. Particularmente, se problematiza su empleo en el contexto universitario, dados los resultados surgidos en los dos grupos focales realizados con docentes en formación que cursan la carrera de Educación Básica, en una universidad chilena de la Región Metropolitana, Santiago, Chile. La necesidad de incorporar este debate en la formación docente y en la comunidad universitaria permite, en primer lugar, discutir en torno al modo en que es evaluado el uso del lenguaje inclusivo; en segundo término, contribuye a ampliar las perspectivas de cómo se lee la realidad; y, finalmente, aporta a revisar el modo en que se nos ha construido desde lo lingüístico. En definitiva, el lenguaje inclusivo ya existe y en su uso está implicada la identidad de las personas.

Palabras clave: formación docente, identidad, lenguaje inclusivo.

\section{Gender-neutral language as an empathy and identity "norm": a future teachers and professors' discussion}

\begin{abstract}
The paper provides an insight into gender-inclusive language. It focuses on how gender-inclusive language is used in higher-education contexts. The paper analyses the data obtained from focus groups with teacher-training students from a university in the Metropolitan Region area (Santiago, Chile). Firstly, the incorporation of this debate into the teacher-training program and the university community itself furthers the discussion on how gender-inclusive language is viewed. Secondly, it helps widen the perspectives with which the reality is understood. Thirdly, it contributes to review the way people have been culturally constructed. In short, gender-inclusive language does exist and people's identities are implied in its use.
\end{abstract}

Una versión inicial de este trabajo fue presentada como ponencia durante el workshop sobre lenguaje inclusivo, realizado el 14 de mayo de 2019 en la Universidad Católica Silva Henríquez, UCSH. Las autoras dan Fentren mañum (muchas gracias) a todes quienes participaron en los nütramkawün (conversatorios) que dieron curso a estas primeras reflexiones.

Chilena. Doctora en Educación, Universidad Católica Silva Henríquez, UCSH, Santiago, Chile. scastillos@ucsh.cl

*** Chilena. Magíster en Educación, Universidad Católica Silva Henríquez, UCHS, Santiago, Chile. jmayo@ucsh.cl 
Keywords: Gender-inclusive language, Identity, Teacher training.

\section{Llitun (inicio)}

El debate en torno al lenguaje inclusivo ha tomado mayor fuerza y profundidad el último tiempo, producto de las discusiones de las organizaciones sociales feministas y de mujeres que han vuelto a cuestionar el sentido común de nuestra sociedad. Si bien la discusión acerca de cómo incorporar a todas las identidades en nuestra habla ya estaba presente y entregando propuestas - que el status quo del español ha rechazado-, esta se ha profundizado en el último año gracias a diversas intervenciones de los espacios sociolingüísticos públicos y privados.

Ahora bien, una discusión de este tipo no es solo de carácter lingüístico, como muchos y muchas han afirmado, sino que se trata de un debate en la esfera de lo social, de lo sociolingüístico, lo político y cultural (Barker \& Galasinski, 2001; Butler, 2004; Foucault, 1977). En esta línea, también corresponde a una discusión que recientemente se ha situado en el marco de los derechos humanos de las personas que no se sienten parte del binomio "a-o" pues, como afirma Jiménez y Román (2011):

El lenguaje no es una mera herramienta mediante la cual expresamos y comunicamos nuestros pensamientos. El lenguaje hace pensamiento, se piensa cuando se habla y, al mismo tiempo, representa y construye realidad. Es el sentido y medio central mediante el cual entendemos el mundo y construimos la cultura (p. 175).

El uso de la '@', de la 'x' y de la vocal 'e' tiene estrecha relación no con la lengua per se, sino que con las personas que hacen uso de esta. El empleo de una lengua en una sociedad tiene un componente de poder y quienes ostentan ese poder deciden qué lengua se usará y cómo. A pesar de esto, los sectores minoritarios históricamente se han manifestado con el propósito de tener representación en lo sociolingüístico: los pueblos indígenas levantando y recuperando el uso de sus lenguas ${ }^{1}$, las identidades territoriales relevando el uso de su variedad lingüística y,

En este texto hemos decidido incorporar conceptos del mapudungun (lengua mapuche) que nos ayudan a explicar nuestros argumentos desde otra perspectiva. 
en la misma línea, las identidades de género exigiendo visibilidad en el habla cotidiana.

Hemos querido reflexionar en torno a esto desde un territorio en específico que creemos es un espacio relevante al momento de plantearse una transformación social o, al menos, encaminarse hacia ella: el territorio de la educación, particularmente la formación docente. ¿Por qué? La inquietud respecto del lenguaje inclusivo está presente en los diarios, en la televisión, en las redes sociales, en las movilizaciones, en las calles, en la academia, en la escuela y en los organismos públicos, entre tantos otros espacios. Sin embargo, la controversia en torno al uso de esta propuesta sociolingüística tiene que ver con un aspecto "normativo" y con instituciones/personas a cargo de esta norma. Dentro de estas instituciones, la escuela es una de ellas y, entre las personas, el profesorado también personifica esta norma.

Si revisamos los prejuicios existentes en torno al lenguaje inclusivo, uno de los primeros es la idea de la deformación del lenguaje, esto es, la concepción de que la lengua es un ente rígido y sin conexión con los cambios sociohistóricos. Creemos que desde la escuela y la formación docente es posible comenzar a debatir frente a esta propuesta, ya que nos situamos en un espacio normado y nos relacionamos con estudiantes que, en el futuro, serán quienes representen dicha norma. En este sentido, dialogar en torno al lenguaje inclusivo con quienes enseñarán luego la lengua española a las futuras generaciones nos parece un espacio pertinente e interesante para iniciar la discusión de esta propuesta.

De tal manera, al hacernos la pregunta por el lenguaje inclusivo y las tensiones que también nos cruzan como formadoras de formadores, pensamos en indagar acerca de qué piensan los docentes en formación en relación con su uso, cuáles son sus ideas y cómo lo tratarían con sus futuros estudiantes.

En términos metodológicos, realizamos dos grupos focales con docentes en formación que cursaban la carrera de Educación Básica, en una universidad de la Región Metropolitana, Santiago, Chile. Para empezar a reflexionar respecto de este tema de manera introductoria, el propósito fundamental de esta conversación radicó en la oportunidad de poner en discusión el uso de lenguaje inclusivo en la comunidad universitaria, abriendo un espacio que, según la voz de estudiantes, "si ha salido la discusión, ha salido desde las mismas estudiantes y la hemos 
compartido entre nosotras mismas; en los espacios de clases no se ha dado la instancia" (B. C., comunicación personal, mayo 2019).

Así, los grupos focales iniciaron con un sencillo ejercicio cuya instrucción fue elaborar un título de prensa para una nota periodística que contemplara los siguientes elementos:

1. Consigna: A partir de los siguientes elementos, redacte un posible título para una nota informativa de prensa:

- $\quad$ Actividad: visita al museo.

- $\quad$ Participantes: Simona, Javiera, Rayén, Valeska, Claudio y Catalina (12 años).

- Contexto: espacio escolar.

Al revisar los títulos elaborados en ambos grupos fue posible apreciar que hubo una preocupación por escribir encabezados que consideraran al grupo completo de participantes de la actividad. En todas las respuestas, el uso del sustantivo estudiante o adolescente dio inicio a la oración, a pesar de que solo había un sujeto masculino. A continuación se presentan algunos de los titulares producto de la actividad:

Estudiantes de la escuela Gabriela Mistral visitan el Museo Histórico Nacional para conocer los hechos concretos históricos.

Siete estudiantes visitan museo.

Estudiantes de doce años realizan visita a Museo de Derechos Humanos.

Estudiantes de sexto año visitan por primera vez el Museo de la Memoria y los Derechos Humanos.

Adolescentes obtienen una salida pedagógica al Museo de Historia Natural.

Estudiantes de segundo año visitan el museo.

Siete estudiantes de sexto básico desaparecen en una visita de museo.

Niñas y niños hacen visita a Museo de la Memoria.

Seis estudiantes de doce años participan de salida pedagógica al museo.

Esta tarea no solo constituyó un desafío como formadoras de formadores, sino que fue útil para situarnos en torno a qué formas y recursos son usados por los docentes en formación para dar cuenta de grupos diversos. De igual modo, nos permitió testear de manera inicial si esa preocupación estaba presente y cómo se resolvía en un ejercicio 
simple. Así, a partir de este ejercicio dimos comienzo a las preguntas y al debate en lo que llamamos nütramkawün (conversatorio).

\section{Nütramkawün: cómo pensamos opciones sociolingüísticas inclusivas desde la formación docente}

Los grupos focales realizados con docentes en formación dejaron ver dos posturas respecto de la propuesta del lenguaje inclusivo: por un lado, pudimos observar una gran preocupación en torno a la manera en que los estudiantes de Pedagogía pueden usar el lenguaje inclusivo en la universidad y en las escuelas donde realizan sus prácticas; y, en segundo lugar, apreciamos una postura más desinteresada que consideraba la propuesta inclusiva como una imposición.

Tomando en cuenta la experiencia generada en los grupos focales, planteamos el siguiente punto de vista: es necesario promover la discusión sobre el uso de lenguaje inclusivo en la comunidad universitaria.

Un primer argumento para ello encuentra asidero en el modo en que es evaluado el empleo del lenguaje inclusivo en el contexto universitario. $\mathrm{Al}$ respecto, el discurso del estudiantado revela que su uso - en algunas ocasiones - es sancionado, restándole décimas al puntaje final obtenido, como se señala a continuación:

Seguimos utilizando la 'o', la 'e', o en los trabajos, por ejemplo, es como, normalmente pongo estudiantes, docentes, aprendices, como 'las y los' alumnos, aunque no debería, porque uno no puede poner 'las y los', porque es la misma palabra, solamente que cambia la consonante (por la repetición lingüística), pero igual es importante, o sea, hay profesores que sí te bajan puntos si uno pone 'las y los', pero es algo que igual uno debería tener presente (J. C., comunicación personal, mayo 2019).

Aunque la constatación de este hecho no dialoga con la idea de que las lenguas son sensibles a los cambios extralingüísticos (Furtado, 2013), es evidente que dichos cambios tropiezan con actitudes de resistencia, sobre todo, en contexto formales. En estos espacios, lo habitual es que la norma se instale y aquello que la transgrede sea percibido en términos perjudiciales. Para del Valle (2018), se trata de "la política de la incomodidad" (p. 21) que nos dispone negativamente frente al cambio, en particular, cuando el acto de habla se expresa en situaciones 
comunicativas altamente codificadas: no respetar la gramática de la lengua es caer en el incumplimiento de las reglas que la constituyen.

Esta idea de la norma como "camisa de fuerza" ya lo expresaba Coseriu (1973), al referirse a esta como aquello que restringe la libertad expresiva y limita las posibilidades de usos lingüísticos, puesto que es concebida como un "sistema de realizaciones obligadas, de imposiciones sociales y culturales" (p. 98). El descuento de décimas en los puntajes, entonces, responde a una sanción de la norma, la que provoca la fosilización del lenguaje y desconoce que este es desarrollado por el ser humano y, como tal, debiera adecuarse a las necesidades y a las estructuras de la sociedad.

Cuando aún no está instalada la discusión en torno a la relación lenguaje-sociedad, es complejo pensar en modificar la lengua para que se ajuste de mejor manera a una realidad social que experimenta cambios constantes, lo cual se ve reflejado en la actitud que asume la academia al momento de enfrentarse al uso de lenguaje inclusivo por parte de sus estudiantes. En este sentido, podrían ser dos las explicaciones a esta postura: la lengua no se concibe como un espacio de disputa de derechos sociales y, por otro, la morfología del español cuenta con los recursos lingüísticos necesarios para evitar lo artificioso. Ejemplo de esto último, de acuerdo con Guerrero (2019), es el empleo de sustantivos colectivos y abstractos (profesorado por profesores), palabras no marcadas como el caso de los sustantivos comunes (docente) y algunos pronombres (alguien, nadie), adjetivo (brillante) y determinantes invariables (cada, tal); epicenos (víctima, personaje); omisión de expresiones masculinas innecesarias (delante de los sustantivos comunes en cuanto al género, mantenimiento solo de las terminaciones verbales usando estructuras impersonales, modificando de voz activa a pasiva, o bien, utilizando expresiones metonímicas, entre otras); uso de perífrasis (el personal administrativo por administrativos; la bancada política por los políticos); desdoblamientos o duplicaciones, a pesar de la crítica (niños y niñas); aclaraciones, es decir, incorporación de una frase explicativa que clarifique la mención tanto de hombres como mujeres; alternativas gráficas como el caso de las barras (niños/ as) o los paréntesis.

Aunque son diversas las alternativas al masculino genérico que Guerrero (2019) propone, algunas de estas continúan aludiendo al carácter binario de la lengua, con escasa o nula cabida al amplio espectro 
de identidades de género, como el caso de quienes se construyen así: intersex ${ }^{2}$.

Lo cierto es que la norma se configura socialmente, por tanto, decidir desobedecer la matriz gramatical del idioma supone una postura política en la medida en que se hace visible una posición social y situada, de acuerdo con del Valle (2018).

Siguiendo la misma línea argumentativa y a propósito del debate en los grupos focales realizados, el segundo de los argumentos que sustenta la necesidad de fomentar la discusión y reflexión respecto del empleo de lenguaje inclusivo en el contexto universitario se vincula con la posibilidad de ampliar los ángulos de construcción de conocimiento. En este sentido, el desconocimiento lleva a reproducir un discurso que configura la realidad desde una sola mirada, marginando la posibilidad —en este caso - de cuestionar la masculinización del lenguaje. Desde esta perspectiva, en uno de los grupos focales se señaló:

yo siento que igual nosotras como una generación nueva que vamos a salir, podemos insertar eso y cambiar el lenguaje, porque es sumamente fundamental que nosotras lo trabajemos desde muy pequeños, porque los chicos, ya como yo le estaba contando denante, desde ya quinto empiezan a ser machos (C.C., comunicación personal, mayo 2019).

En relación con el punto anterior, Bengoechea (2019) manifiesta la urgencia de debatir en torno a la obligación, necesidad y posibilidades del lenguaje inclusivo, puesto que contribuiría a desmantelar la estructura

\footnotetext{
2 Según el Programa de Naciones Unidas “Libres \& Iguales”:

Las personas intersex nacen con caracteres sexuales (como los genitales, las gónadas y los patrones cromosómicos) que no se corresponden con las típicas nociones binarias sobre los cuerpos masculinos o femeninos. Intersex es un término que se utiliza para describir una amplia gama de variaciones naturales del cuerpo. En algunos casos, los rasgos intersex son visibles al nacer, mientras que en otros no se manifiestan hasta la pubertad. Algunas variaciones cromosómicas de las personas intersex pueden no ser físicamente visibles en absoluto.
}

Según expertos, entre un $0,05 \%$ y un $1,7 \%$ de la población nace con rasgos intersex; el porcentaje que representa el umbral superior es similar al número de personas pelirrojas.

Ser intersex está relacionado con las características biológicas del sexo y no tiene que ver con la orientación sexual o la identidad de género de las personas. Una persona intersex puede ser heterosexual, gay, lesbiana, bisexual o asexual, y puede identificarse como mujer, hombre, ambos o ninguna de las dos cosas.

Debido a que sus cuerpos son percibidos como diferentes, los niños y adultos intersex son a menudo estigmatizados y sometidos a múltiples violaciones de sus derechos humanos, incluyendo violaciones de sus derechos a la salud y la integridad física, a no ser objeto de tortura ni de malos tratos, a la igualdad y la no discriminación. (Naciones Unidas, 2018) 
social patriarcal, presente en el uso excesivo del género masculino como señal de representación totalitaria, ya que construir la realidad desde un solo prisma tiene implicancias teóricas y prácticas significativas. Ejemplo de ello es la inequidad histórica entre mujeres y hombres que, si bien no se resuelve a partir de una decisión lingüística, examinar la historicidad del lenguaje - al menos - permite abrir el tema y comprender que este configura la realidad y que no está exento de las desigualdades que acontecen en ella.

Si bien el género en español se expresa de diversas maneras, entre ellas como binomio (aunque no solamente así), lo cierto es que se caracteriza por su carga sexuada (Minoldo y Cruz Balián, 2018). Esto, tal como ya se mencionó, remite a una realidad que da cuenta de las asimetrías de género y de las construcciones de sentido que se configuran en torno a estas.

Lo anterior tiene evidencias empíricas en los resultados de diversos estudios mencionados por Minoldo y Cruz Balián (2018): el experimento de Phillips y Boroditski (2003), demostró la relación entre el nombre del objeto y su género gramatical; "Sera y su equipo reveló que el género gramatical de los objetivos inanimados afecta las propiedades que les hablantes asocian con esos objetos" (p. 10); y el estudio realizado por Segel y Boroditsky (citado en Minoldo y Cruz Balián, 2018) afirman que existe influencia del género gramatical en la construcción de ideas abstractas: "la elección de una figura masculina o femenina puede predecirse por el género gramatical de la palabra en la lengua nativa del artista" (p. 10).

Los experimentos referidos permiten volver a la idea de Sapir (1986) y Whorf (1971), quienes advirtieron el profundo vínculo entre lenguaje y pensamiento. De ahí, entonces, la necesidad de develar aquello que oculta el uso del lenguaje, porque en él se afirman y negocian no solo las identidades sociales de quienes interactúan, sino también sus sentidos singulares (Narvaja de Arnoux y del Valle, 2010). Lo anterior no es muy distinto de lo que hasta aquí se ha venido discutiendo, esto es, problematizar el uso de lenguaje inclusivo en la comunidad universitaria permite develar-nos cómo hemos sido construidos. Esto implica detenerse y revisar los modos en que configuramos la realidad a partir del lenguaje, a fin de cuestionar las relaciones de poder y las negaciones implícitas en su uso. 
$\mathrm{Al}$ respecto, es conocida por todos la respuesta que habitualmente solía darse frente a la siguiente pregunta (al menos antes del debate que justifica la escritura de este artículo): ¿cómo saludar a un grupo constituido por mujeres y un solo hombre? Si 'todos' era la respuesta, ¿por qué frente a un grupo conformado por hombres y solo una mujer la respuesta no es 'todas'? ¿Acaso un ejemplo como este no defiende una posición de poder? Sin duda, una explicación que vaya de la mano con un enfoque crítico, no desconoce el modo en que el patriarcado ha permeado el empleo del lenguaje. Custodiar el sistema patriarcal (Muntané, 2018) a través del uso de un lenguaje sexista es continuar reproduciendo las relaciones de poder que han caracterizado a la sociedad.

La lengua como espacio de poder simbólico permite disputar los tipos de relaciones entre los diferentes grupos que conforman la sociedad. Eso es lo que ha venido sucediendo con la eclosión de las demandas feministas, donde los actores ven en la materialización de la lengua un gesto simbólico que contribuye a denunciar abusos contra la mujer y a conquistar derechos que han sido arrebatados, jibarizados e invisibilizados. Es así que, dentro de las conversaciones sostenidas con estudiantes se afirmó, por ejemplo: “a lo mejor, en verdad, hablar con la 'e' no soluciona el problema, pero ayuda a que vayamos dejando de lado esta marginalidad del lenguaje, porque el lenguaje construye realidades" (A. P., comunicación personal, mayo 2019).

En concordancia con lo anterior, nadie cuestiona expresiones como la siguiente: “Aquello que no se nombra no existe" y si el lenguaje configura la realidad, ¿por qué continuar naturalizando los efectos de la dominación masculina y no avanzar hacia la deconstrucción de tales patrones heredados? Un posicionamiento como este exige proponer nuevas categorías para representar la realidad en la que se habita (Minoldo y Cruz Balián, 2018) con el propósito de dar cabida, desde el plano lingüístico, a los grupos que han sido sostenidamente marginados.

Si bien el uso de algunas alternativas al masculino genérico planteado por Guerrero (2019) aporta a desmoronar el lugar de privilegio que ha ocupado el empleo de este genérico, ello no resuelve, en su dimensión más abarcadora, los problemas de desigualdad lingüística. Se visibiliza la inequidad histórica entre mujeres y hombres, pero esta propuesta de carácter binario no da respuesta a las identidades emergentes. La opción más plausible es la que puede apreciarse en Giacchetta (2018): 
Me imagino lo inmensamente reparador que es para una identidad diversa como la mía, o incluso para otras múltiples realidades - como las de las personas intersexuales—, ingresar a un lugar donde los reciben con un - bienvenides, autorizándolos a expresar su género sin condicionantes y estándares que no fueron pensados para todes. Y lo liberador que es no tener que dar explicaciones sobre la propia presencia, porque en definitiva todes buscamos lo mismo: existir (p. 16).

El uso de la letra 'e' permite una clara concesión a la diversidad de género, contribuyendo a derribar asimetrías lingüísticas para construir una sociedad más equitativa y menos violenta.

Sin embargo, la argumentación que se ha venido hilvanando en torno a la necesidad de promover el debate sobre el uso de lenguaje inclusivo en la comunidad universitaria podría no significar una preocupación generalizada en estos contextos, sobre todo, si son escasas las instituciones de educación superior que cuentan con documentos orientados a abordar un lenguaje no sexista. Al respecto, actualmente algunas de las instituciones chilenas que cuentan con manuales de lenguaje inclusivo son el Ministerio de la Mujer y de Equidad de Género, el Ministerio de Educación, la Comisión Nacional de Ciencia y Tecnología, el Ministerio de las Culturas, las Artes y el Patrimonio, el Ministerio de Agricultura, el Instituto Nacional de Estadísticas y la Corporación Nacional del Cobre, entre otras. No obstante, aún no se cuenta con universidades que hayan incorporado un manual que aborde estas temáticas dentro de sus espacios de formación.

De acuerdo con López (2018), el uso del lenguaje inclusivo avanza raudamente entre los jóvenes y son las redes sociales las que atestiguan este movimiento, pero ¿qué cabida tiene en los contextos más formales cuando - al parecer- en estos se advierte mayor resistencia? No es extraño pensar que el respeto a la norma es más habitual en estos espacios. Ejemplo de ello es la postura que la propia Real Academia Española (RAE) ha manifestado en relación con el uso del lenguaje inclusivo. Apegado a las reglas de la lengua, la entidad sostiene que

el uso del @, la letra E y la X, como supuestas marcas de género inclusivo, es ajeno a la morfología del español, además de innecesario, pues el masculino gramatical, ya cumple esa función como término no marcado de la oposición de género (Cooperativa, 2018, párr. 3). 
Situación similar se advierte en el discurso de la lingüista Concepción Company (2017), quien afirma que hacer visible la igualdad de género puede derivar en el empobrecimiento de la lengua; más aún, trastocar la gramática de un idioma conlleva el riesgo de transgredir su memoria histórica, de modo que forzar sus mecanismos morfológicos, por consiguiente, es no comprender que en gramática el género no corresponde al sexo.

En esta línea, dentro de los grupos focales se manifestó la necesidad de seguir la norma, por ejemplo, se afirmó:

yo creo que también debiésemos buscar recursos teóricos que avalen este, el lenguaje inclusivo y que no sea así como netamente para atender la diversidad y todo el tema, sino que tenga algo teórico, tenga algo contundente como un argumento para defenderlo (P. P., comunicación personal, mayo 2019).

En la misma línea y apelando a lo normativo como "democrático" se manifestó:

sí y que la formación docente se defienda con un argumento teórico, que valide también la implementación en todos, por eso digo, es en todos los estudiantes de educación básica, porque de lo contrario, de no suceder así, sería como una imposición arbitraria, de lo que al parecer para unos pocos es correcto llevar a la práctica, ahora si hay un sustento teórico, ya deja de ser arbitrario, entonces sería más democrático (P. P., comunicación personal, mayo 2019).

Ahora bien, ¿cómo nos sintonizamos con quienes poseen la regla, la norma, desde lo teórico; ¿cómo alinearse por ejemplo con la RAE si en 2017 definía al sexo débil como el “conjunto de mujeres” y recién en 2018 modificó la definición de fácil, puesto que en una de sus acepciones (5) se refería a "dicho especialmente de una mujer. Que se presta sin problemas a mantener relaciones sexuales" (El Desconcierto, 2018, párr. 5). De igual manera, el pasado año publicó que la voz 'feminazi' es un acrónimo de 'feminista' + 'nazi'. Respondiendo la pregunta de una usuaria, declaró que dicha palabra se utiliza despectivamente con el sentido de "feminista radicalizada" (El Desconcierto, 2018, párr. 2). 
La expresión "Usted no lo diga"3 del controversial profesor Banderas no dialoga con estos tiempos, pues la realidad social exige problematizar el uso del lenguaje. Si bien, como se ha declarado, el género gramatical no corresponde al sexo, ¿cómo se explican los resultados de los diversos estudios mencionados por Minoldo y Cruz Balián (2018), que afirman -

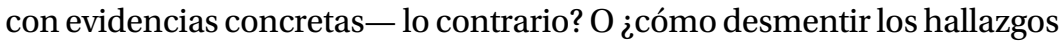
de la investigación de Dies Vervekjen (Citado en Minoldo \& Cruz Balián, 2018)mencionados por los mismos autores que revela, en entornos de habla de alemán y neerlandés, la influencia de las formas lingüísticas en las percepciones de niños y niñas acerca de trabajos estereotipadamente masculinos?

\section{Feymew... (entonces)}

Lo cierto es que el lenguaje inclusivo ya existe, se usa, está incomodando en diversos espacios y si incomoda es porque hay una necesidad de visibilizarse en lo más básico, en lo más cotidiano y a la vez en lo más poderoso en la sociedad: el lenguaje. Como se señaló en uno de los grupos focales con estudiantes, "entonces es un tema que se tiene que educar, se tiene que trabajar porque conlleva más allá de un cambio de letra, conlleva la identidad de cada persona" (B. C., comunicación personal, mayo 2019).

De tal forma, es necesario abrir la discusión en espacios donde la lengua es la herramienta principal, donde se enseña y se usa para enseñar. Así, resulta fundamental volver sobre los universales lingüísticos, sobre la arbitrariedad del lenguaje y su poder creativo con el objetivo de profundizar en los prejuicios que se hallan en el sentido común y abrir paso a nuevas formas que sean capaces de sumar voces.

En este artículo ensayístico hemos querido iniciar un debate que incluso a nosotras mismas como docentes nos tensiona, y que desde el uso de otras lenguas, como el mapudungun, graficamos utilizando palabras sin género para saltarnos la incomodidad. En la misma línea, nos planteamos como proyecciones de esta primera reflexión, dar continuidad a estas discusiones en el ámbito universitario de manera transversal y avanzar en el diseño de un manual de lenguaje inclusivo que recoja estas inquietudes en el contexto de nuestra institución.

Ver referencia en http://terceracultura.cl/2010/11/usted_no_me_diga/ 
De tal modo, esta es una primera ventana para dar paso a discusiones mayores dentro del estudiantado en la formación docente, donde tal vez la respuesta al problema sea mucho más sencilla y simplemente implique abrirse al uso de estas formas hasta que se conviertan en norma.

Por ahora, el nütramkawün sostenido con docentes en formación problematiza el uso de lenguaje inclusivo y la necesidad de incorporar el debate en el contexto universitario, debido a que permite discutir acerca del modo en que es evaluado su empleo; contribuye a ampliar las perspectivas de cómo se lee la realidad; $\mathrm{y}$, por último, aporta a revisar el modo en que se nos ha construido como personas.

\section{Referencias bibliográficas}

Barker, C. \& Galasinski, D. (2001). Cultural studies and discourse analysis: A dialogue on language and identity. London: Sage.

Bengoechea, M. (2019). Razones de la lingüística feminista para abogar por un lenguaje inclusivo. En M. Bengochea (Directora). El lenguaje inclusivo: un debate en torno a su obligación, necesidad y posibilidades. Simposio llevado a cabo por Centro de Estudios Políticos y Constitucionales en colaboración con Clásicas y Modernas, Madrid.

Butler, J. (2004). Lenguaje, poder e identidad. Madrid: Síntesis.

Cooperativa (15 de junio de 2018). ¿Lenguaje inclusivo? RAE se refirió al uso de las letras $E$ y $X$ en el debate de género. Recuperado de https://www.cooperativa.cl/noticias/sociedad/sucesos/ lenguaje-inclusivo-rae-se-refirio-al-uso-de-las-letras-e-y-xen-el/2018-06-15/163125.html

Coseriu, E. (1973). Sistema, norma y habla. Teoría del lenguaje y lingüística general. Madrid: Gredos.

Company, C. (3 de diciembre de 2017). Debate urgente: ¿tienen arreglo los machismos del castellano? Clarín. Recuperado de https:// www.clarin.com/cultura/debate-urgente-arreglo-machismoscastellano 0 Hy3aMI1Zz.html

Del Valle, J. (2018). La política de la incomodidad. Glotopolítica. Recuperado de https://glotopolitica.com/2018/08/21/ la-politica-de-la-incomodidad/ 
"Feminista radicalizada": La RAE recibe numerosas críticas tras definir en Twitter la palabra "feminazi" (24 de agosto de 2018). El Desconcierto. Recuperado de https://www.eldesconcierto. cl/2018/08/24/feminista-radicalizada-la-rae-recibenumerosas-criticas-tras-definir-en-twitter-la-palabrafeminazi/

Foucault, M. (1977). Arqueología del saber. México, DF: Siglo XXI.

Furtado, V. (2013). El lenguaje inclusivo como política lingüística de género. Revista Digital de Políticas Lingüísticas, 5, 48-70. Recuperado de https://revistas.unc.edu.ar/index.php/RDPL/ article/view/8656/9513

Giacchetta, C. (11 de agosto de 2018). Damas y caballeros, presentamos para 'todes': ¡el lenguaje inclusivo! La Voz. Recuperado de https://www.lavoz.com.ar/opinion/damas-y-caballerospresentamos-para-todes-lenguaje-inclusivol

Guerrero, S. (2019). Alternativas al masculino genérico. En M. Bengochea (Directora). El lenguaje inclusivo: un debate en torno a su obligación, necesidad y posibilidades. Simposio llevado a cabo por Centro de Estudios Políticos y Constitucionales en colaboración con Clásicas y Modernas, Madrid.

Jiménez y Román (2011). Lenguaje no sexista y barreras a su utilización. Un estudio en el ámbito universitario. Revista de Investigación en Educación, 2 (9), 174-183.

La RAE se actualiza: "Fácil” ya no significará "mujer que se presta sin problemas a mantener relaciones sexuales" en el diccionario (14 de marzo de 2018). El Desconcierto. Recuperado de https:// www.eldesconcierto.cl/2018/03/15/la-rae-se-actualiza-facilya-no-significara-mujer-que-se-presta-sin-problemas-amantener-relaciones-sexuales-en-el-diccionario/

López, V. (12 de junio de 2018). 'Todes les diputades': el lenguaje inclusivo avanza entre los jóvenes y genera polémica. Clarín. Recuperado de https://www.clarin.com/sociedad/todes-diputadeslenguaje-inclusivo-avanza-jovenes-generapolemica 0 Sy6mQt6em.amp.html

Minoldo, S. y Cruz Balián, J. (2018). La lengua degenerada. En El gato y la caja. Recuperado de https://elgatoylacaja.com.ar/ la-lengua-degenerada/ 
Muntané, I. (15 de agosto de 2018). El lenguaje es política. En El País. Recuperado de https://elpais.com/elpais/2018/08/07/ opinion/1533666589 152469.html

Naciones Unidades (2018). Intersex. Recuperado de https://www.unfe. org/wp-content/uploads/2018/10/Intersex-ES.pdf

Narvaja de Arnoux, E. y del Valle, J. (2010). Las representaciones ideológicas del lenguaje. Discurso glotopolítico y panhispanismo. Ideologías Lingüisticas y el Español en Contexto Histórico, 7(1), 1-24. https://doi.org/10.1075/sic.7.1.01nar

Sapir, E. (1986). El leguaje. Introducción al estudio del habla. Madrid: Ediciones Fondo de Cultura Económica.

Whorf, B. (1971). Lenguaje, pensamiento y realidad. Barcelona: Barral. 